\title{
Follow up of an immunocompromised contact group of a case of open pulmonary tuberculosis on a renal unit
}

F A Drobniewski, J Ferguson, K Barritt, R M Higgins, M Higgon, D Neave, A H C Uttley, D O'Sullivan, A Hay infectious cause of morbidity and mortality worldwide, infecting eight million and killing three million people annually. ${ }^{12}$ The decline in the number of cases of clinical tuberculosis in the developed and parts of the developing world ceased or reversed in the mid 1980s and the reasons for this are discussed in detail elsewhere. $^{12}$

Tuberculosis in immunosuppressed patients, including those undergoing renal transplantation, is associated with atypical clinical and radiographic presentation, rapid disease progression, and a high mortality. Tuberculosis may be relatively common in renal patients for example, it has been diagnosed in up to $28 \%$ of renal patients undergoing maintenance dialysis in one centre. ${ }^{3}$ In two recent studies and numerous case reports incidences of tuberculosis of $1 \cdot 7-3 \cdot 5 \%$ in renal transplant centres in the United Kingdom and in Saudi Arabia are reported; geographical and ethnic variation are reflected in the incidence of $11.5 \%$ reported in India. ${ }^{4}$ These studies confirm the susceptibility of some renal patients to tuberculosis compared with the general population, that disseminated tuberculosis is more common after renal transplantation, that it frequently requires invasive procedures for diagnosis, and often leads to loss of the graft when immunosuppressive therapy is discontinued. ${ }^{45}$

\section{Methods}

In September 1993 a staff member working part time in the Renal Unit and Regional Transplant Centre of a London hospital was diagnosed with smear positive cavitating pulmonary tuberculosis. Relevant past medical history of the index case included BCG vaccination in 1974 and a domestic tuberculosis contact in 1988 which had been followed up appropriately; Heaf testing at that time was negative and BCG vaccine was administered. A subsequent pre-employment health screen in 1990 was unremarkable. No Heaf test was performed. The index case, a smoker, had had pulmonary symptoms for most of the preceding six months and was subsequently referred to a chest physician who diagnosed tuberculosis clinically, confirmed by cavitating lesions on chest radiography. Sputum was smear positive and yielded a fully susceptible strain of $M$ tuberculosis. The staff member was successfully treated with conventional combination antituberculous chemotherapy. 1 January 1993 was
Mycobacterial diseases are a major health problem in the world today. Tuberculosis due to Mycobacterium tuberculosis is the predominant 
designated as the onset of potential infectious exposure because of the long duration of symptoms including productive cough and the advanced changes on chest radiography.

\section{ACTION AND ORGANISATION}

An Emergency Control of Infection Committee (EICC) chaired by the hospital infection control doctor was established which included the local consultant in communicable disease control and representatives from the renal, respiratory, public health, microbiological, radiological, and occupational health departments, together with representatives from the management of the Trust. Its role was to define the protocol to be followed (through a smaller Clinical Protocol Group), establish the extent and end point of the investigation, disseminate information to all relevant parties including the media, and to undertake major financial and managerial decisions. Designated additional telephone lines for patient advice supported by medical advisors and information sheets on tuberculosis were installed. Patients were interviewed using a standardised questionnaire either by a renal nurse dedicated to the contact group, a chest unit liaison sister, a clinical microbiology registrar, an infection control nurse or an occupational health advisor as appropriate. Screening chest radiographs were reviewed initially by staff in the departments of renal or chest medicine or clinical microbiology, but were all subsequently evaluated by a consultant radiologist or chest physician. All patients or staff with suspicious symptoms or chest radiographs were referred to the chest clinic for further evaluation.

A small Data Management Group (DMG) was established comprising a registrar in clinical microbiology, senior registrar in public health,

Patients:

- All contacted by letter, explanation, invitation for screening

- At first visit:

Symptom questionnaire, BCG status/scar and chest radiography

Heaf test for immunocompetent patients only

Chemoprophylaxis with isoniazid if necessary

All chest radiographs reviewed by chest consultant

Review by chest clinic if necessary

- At follow up:

Renal $\Rightarrow$ Questionnaire

Chest radiography

Surgical $\Rightarrow$ Reassurance

GP letter

8

Chest clinic if required

Staff:

- Fact sheet and invitation for screening

- Occupational health consultation:

Symptoms questionnaire

Heaf testing

Chest radiography

Chest clinic referral if necessary

- Former staff: local consultants in communicable disease control notified of contact chest liaison sister, renal nurse, infection control nurse, occupational health advisor and information officer. Its role was to co-ordinate data collection, organise follow up of the surgical patient group, identify possible tuberculosis cases, and exclude tuberculosis as a cause of death in those who died before screening began. Case records of deceased individuals, death certificates, laboratory reports and tuberculosis notifications were evaluated to exclude tuberculosis as a cause of death in the study population. The consultants in communicable disease control of areas in which former Trust staff members were now working were contacted to follow up staff using the BTS guidelines. ${ }^{6}$ Repeat letters were sent to patient and staff non-attenders and subsequently to their GPs. In all cases GPs were informed of their patient's exposure, the result of the screening or lack of attendance, and helpline numbers and advice were offered.

The cost of screening was derived from individual departmental costs which were assessed by appropriate members of the EICC and the costs of meetings which were costed on the basis of the number of hours spent by individuals using mid point salary scales of the Whitley Council for 1993-4 including $14 \%$ on costs.

\section{CONTACTS}

Contacts were defined using the BTS guidelines ${ }^{6}$ and formed four groups: (1) family contacts; (2) renal patients; (3) surgical patients; and (4) staff. All family contacts were followed up locally in accordance with BTS guidelines. No cases of clinical disease were recorded but two children were placed on six months isoniazid chemoprophylaxis. This study is concerned with the last three groups.

The follow up period was initially set at two years ${ }^{6}$ (later modified), with the first follow up date at three or six months since last contact and then at six or 12 months, 18 months, and 24 months. The lengthy follow up period was felt necessary as diagnosis was likely to be more difficult in immunocompromised renal patients in whom the average age was 54 years, tuberculin testing was unreliable, and in whom there were frequently non-specific pulmonary symptoms with abnormal chest radiographs. Follow up was as described in the figure. Heaf tests were not offered to the renal contacts due to the high false negative rate in this older and immunosuppressed group.

Chemoprophylaxis with isoniazid $200 \mathrm{mg}$ (plus $10 \mathrm{mg}$ pyridoxine) was given for six months to those identified as being at special risk-namely, patients transplanted or receiving immunosuppression for rejection, patients who were HIV positive, and those receiving cyclophosphamide as induction therapy for renal vasculitides.

Surgical contacts were followed up as indicated in the figure but they were reviewed in two additional clinics, Heaf tested as appropriate, and offered chest radiography. Following the initial screen and the lesser risk to these contacts, surgical patients were asked to 
Table 1 Renal contacts of index case screened in accordance with the protocol outlined in the figure

\begin{tabular}{lcc}
\hline & First screening & Second screening \\
\hline Requiring screening & 203 & \\
Screened in district of residence & 23 & $11(5 \cdot 6 \%)$ \\
Died before screening & 52 & 217 \\
Requiring screening locally & 228 & 196 \\
Screened in study & 220 & $4(1 \cdot 8 \%)$ \\
Signed disclaimer & $5(2 \cdot 2 \%)^{\mathrm{a}}$ & $7(3 \cdot 2 \%)$ \\
Did not attend & $3(1 \cdot 3 \%)$ & $95 \%$ \\
\% screened & $97 \%$ & \\
\hline
\end{tabular}

${ }^{a}$ Figures in brackets represent percentages of contacts requiring screening locally ( $n=228$ at first screening, $\mathrm{n}=217$ at second screening).

Table 2 Symptoms, BCG history, and screening undertaken among different contact groups ( $n=$ number completing all or part of the screening process)

\begin{tabular}{lccc}
\hline & $\begin{array}{l}\text { Renal } \\
\text { contacts }\end{array}$ & $\begin{array}{l}\text { Surgical } \\
\text { contacts }\end{array}$ & $\begin{array}{l}\text { Staff } \\
\text { contacts }\end{array}$ \\
\hline No. of questionnaires completed & 220 & 57 & 135 \\
Mean age (years) & 54 & 58 & - \\
Age range & $17-85$ & $18-91$ & - \\
Male & $122(56 \%)$ & $23(40 \%)$ & $36(27 \%)$ \\
Female & $95(44 \%)$ & $34(60 \%)$ & $99(73 \%)$ \\
Symptoms: & $92(42 \%)$ & $24(42 \%)$ & $35(26 \%)$ \\
$\quad$ Cough & $55(25 \%)$ & $17(30 \%)$ & $24(18 \%)$ \\
Phlegm & $3(1 \%)$ & $1(2 \%)$ & $1(1 \%)$ \\
Bloody phlegm & $25(11 \%)$ & $12(21 \%)$ & $4(3 \%)$ \\
Weight loss & $42(19 \%)$ & $6(11 \%)$ & $9(7 \%)$ \\
Fever & $47(21 \%)$ & $13(23 \%)$ & $23(17 \%)$ \\
Sweating & $66(30 \%)$ & $20(35 \%)$ & $107(79 \%)$ \\
BCG history & $217(99 \%)^{\mathrm{a}}$ & $52(92 \%)^{\mathrm{d}}$ & $91(67 \%)$ \\
Chest radiography & $9(4 \cdot 1 \%)^{\mathrm{b}}$ & $0(0 \%)^{\mathrm{c}}$ & $1(0.7)$ \\
Referral to chest clinic & &
\end{tabular}

${ }^{a}$ One patient refused chest radiography at the time of screening, two had chest radiography performed at another hospital.

This group was under regular review by the renal department and decisions regarding chemoprophylaxis/treatment were made without formal referral but in collaboration with the chest plinic.

${ }^{c}$ Although no formal referrals were made, this group was reviewed by a chest liaison sister and Although no formal referrals were made, this group was reviewed
clinical microbiologist with immediate access to the chest clinic.

${ }^{d}$ Of the five patients who did not have screening chest radiographs, one was an inpatient and Of the five patients who did not have screening chest radiographs, one was an inpatient and
another collapsed in the screening clinic with acute pancreatitis and was admitted - both had normal radiographs; three refused radiography.

${ }^{\epsilon}$ No ages were recorded from staff contacts to preserve confidentiality

refer themselves to their GPs if they developed any suspicious symptoms. Written advice to this effect was given to all patients and their GPs and details entered in the surgical case notes.

Staff members with minimal contact were reassured and the remainder followed up by the occupational health department as described in the figure. All 27 family contacts resided outside the district and were followed up by their local contact tracing services.

The protocol was modified by the EICC in the light of proposed changes to the BTS guidelines ${ }^{7}$ that contacts should be followed up for a period of 12 rather than 24 months so that patients and staff who remained symptom free, with no evidence of tuberculosis on chest radiography at 12 months, would be discharged from follow up with notification to their GPs.

\section{Results}

Overall, 576 patient and staff contacts were identified and $85 \%$ (543) were reviewed. Three hundred and three renal contacts were identified including $61(27 \cdot 7 \%)$ with renal transplants, $36(16.4 \%)$ on haemodialysis, 35 $(15.9 \%)$ on ambulatory peritoneal dialysis, and $76(34.6 \%)$ with general nephropathology; 52 patients had died before screening and 23 were screened by their local referring hospital leaving 228 patients for local screening (table 1). A further 11 patients died during the screening process. The mean age, age range, reported BCG status, and proportion screened referred for chest radiography and to the chest clinic are described in table 2. Although 135 (74\%) staff contacts were screened there were significant differences in the groups who attended - for example, only nine (33.3\%) medical staff attended compared with $80.7 \%$ of the other staff groups (nursing, domestic, portering, etc) $\left(\chi^{2} 26.77, \mathrm{p}<0.001\right)$.

The range of symptoms reported among the contacts appears in table 2 . Of the renal contacts, $132(60 \%)$ reported at least one symptom and $85(39 \%)$ two or more. Three patients reported apparent haemoptysis but did not have tuberculosis on further review. Amongst the surgical patients $34(60 \%)$ reported at least one symptom; one individual reported haemoptysis (related to a postoperative problem) and no tuberculosis was detected. Forty five staff $(33 \%)$ reported at least one symptom with one staff member reporting haemoptysis. This employee had ceased employment with the Trust and was followed up by her new employers who concluded that the temporary haemoptysis was due to severe acute bronchitis in a heavy smoker and that there was no evidence of tuberculosis.

Knowledge of BCG vaccination status was variable but staff contacts were generally accurate when status was confirmed by the presence of a BCG scar. Few Heaf test results were available for analysis on patients as no tests were performed on renal patients and many surgical patients were elderly (rendering the test unreliable), or had concurrent illness, or refused to have the test administered or read. Only one staff contact individual with a Heaf grade 3 or 4 had definitely not been vaccinated. Subsequent chest radiography and follow up had not indicated tuberculosis. No other staff gave Heaf tests of grade 3 or 4 . The staff who were Heaf tested were given BCG if negative but continued working if asymptomatic with a prior history of BCG and/or presence of a scar.

The medical records of the 48 staff nonattenders were reviewed indicating that 36 (75\%) had been BCG vaccinated previously. Twenty two $(45 \cdot 8 \%)$ had been Heaf tested during their employment screening with 20 producing a Heaf 1 or 2 reaction and two producing a Heaf grade 3 reaction. Subsequent chest radiography and follow up at the time had not indicated tuberculosis. Screening has been definitely carried out on at least 10 of the 48 individuals $(21 \%)$ who have left the Trust's employment.

\section{OUTCOME}

Sixty three renal patient contacts died during the contact period and between screenings. None of the deaths was believed to be due to tuberculosis following review of case notes, death certificates, or discussion with the attending medical staff or GP. One case of pulmonary tuberculosis was identified during the screening process. A 55 year old white woman receiving haemodialysis who had no symptoms 
Table 3 Final costs associated with the investigation of staff and patient contacts

\begin{tabular}{lcc}
\hline Department or group & \multicolumn{1}{c}{ Cost } & Staff costs as \% of total \\
\hline Emergency Infection Control Committee (EICC) & $£ 2407$ & 100 \\
Clinical Protocol Group & $£ 167$ & 100 \\
Data Management Group (DMG) & $£ 579$ & 100 \\
Renal unit & $£ 6499$ & 92.5 \\
Chest unit & $£ 1143$ & 100 \\
Radiology department & $£ 4445$ & $87 \cdot 1^{\mathrm{c}}$ \\
Occupational health department & $£ 3161$ & $94 \cdot 8$ \\
General management & $£ 693$ & $60 \cdot 8$ \\
Public health and public health laboratory service & $£ 5715$ & $100^{\mathrm{d}}$ \\
Total & $£ 25388$ & \\
\hline
\end{tabular}

${ }^{a}$ Direct costings are derived from mid point salary scales of the Whitley Council for 1993-4 including $14 \%$ on costs. Where costs were estimated for meetings such as those of the EICC only the hourly rate for all those attending was assessed; no allowance was made for travelling time or costs or cancelled clinical sessions.

${ }^{b}$ Apart from meetings a single costing price was used to cover the radiograph, radiographer, and radiologist reporting time.

'An individual package cost of radiograph, radiographer, and radiologist time was provided.

d Photocopying costs and computer facilities were provided from within the routine budget.

and a normal chest radiograph three months after her contact with the index case in October 1993 subsequently reported a productive cough with fever on her second screening in January 1994. She had no other history of contact with tuberculosis, had not been immunised with BCG, and was not on immunosuppressive drugs. She was also hepatitis $C$ positive. A chest radiograph now revealed apical shadowing in both zones consistent with tuberculosis, but cultures of sputum, blood and bronchial washings failed to grow $M$ tuberculosis. A clinical diagnosis of tuberculosis was made, she commenced antituberculous therapy, and has now recovered. In all, 31 immunocompromised renal patients received a course of isoniazid chemoprophylaxis, 18 (16 of whom were transplant patients) at the initial renal unit and 13 transplant patients at a second centre sharing care with this unit.

Tuberculosis was not detected in any of the surgical patients, although several patients reported previous tuberculosis or past exposure and had radiological signs consistent with old calcified tuberculosis. Two pulmonary malignancies were identified incidentally and appropriate referrals made. No deaths from tuberculosis were identified during the follow up period.

\section{COSTING}

The final costs associated with the investigation are given in table 3 . The cost of meetings of the EICC and DMG was $£ 3153$ but the former cost almost five times as much as the latter, reflecting both its larger size and the greater seniority of its members. The overall cost of the study was approximately $£ 25000$ or $£ 44$ per contact screened. Total staff costs were estimated to be $78 \%$ of the total costs.

\section{Discussion}

This study raises several issues regarding the advisability and practicalities of managing a large contact group using the 1990 BTS guidelines. ${ }^{6}$ No guidelines were available for transplant patients and these were formulated during the study. Many of these recommendations appear in the revised 1994 BTS guidelines $^{7}$ in which a less proactive response is advised for all but the immunosuppressed.

Many contacts were identified due to the prolonged period of exposure in a surgical ward and a busy renal unit carrying out approximately 70 transplants per year with 350 transplant patients on follow up, 65 haemodialysis patients, and 135 patients on continuous ambulatory peritoneal dialysis under its care at any one time.

The DMG organised and co-ordinated data collection and reported to the EICC. In practice, although it worked extremely efficiently, it was called upon to make management decisions beyond its original remit. Authority to make day-to-day decisions vested in a small group would have expedited matters, particularly in interpreting BTS guidelines in the context of local conditions.

\section{CONFIDENTIALITY AND EXECUTIVE \\ RESPONSIBILITY}

Inevitably the identity of the index case was known amongst the staff member's peer group but protection of the staff member's identity was considered to be of paramount importance ethically. The organisers of the investigation ensured that confidentiality was preserved, but with complete openness regarding the nature of the disease and the likely risks to patients and staff.

The issue of lead executive responsibility for the outbreak was highlighted by this study. Although a good working relationship existed between the consultant in communicable disease control and the infection control doctor, the exact roles in any similar incident need to be explicitly defined from the outset. In principle, an infection control doctor deals with any outbreak involving the hospital alone, and the consultant in communicable disease control would normally have executive responsibility in any incident involving a notifiable disease in the community or in a serious public health problem originating in a hospital but which affects the population at large. ${ }^{8}$

\section{COSTS AND RESOURCES}

Resources from central Trust funds were made available originally for telephone lines and staffing but costs for additional staff in the key departments involved in the handling of the incident came from their own budgets once anticipated public and media interest did not materialise.

The immunocompromised status of many of the contacts increased costs as a broad range of screening tools had to be deployed. The prevalence of reported symptoms from the questionnaire if used alone, for example, was of low predictive value for both patient groups but was of value in screening staff for tuberculosis. Immunosuppression produces anergy to tuberculin and renal patients frequently had atypical symptomatology and abnormal chest radiography reducing the individual discriminatory power of Mantoux testing, clinical, and radiological examination. 
CONTACTS

Renal contacts were easy to identify as the patients were reviewed regularly and the renal department keeps excellent computerised records which enabled lists to be created quickly. Complete staff lists were also obtained without difficulty. The production of complete lists of surgical patients was surprisingly difficult despite the fact that many of the patients were extra-contractual referrals requiring billing to the originating health authority. It also became clear that, unless a patient dies in hospital, there is no simple mechanism to ascertain which patients have died and so prevent families receiving inappropriate requests for deceased relatives to attend for screening.

The appointment of a dedicated staff nurse in the renal unit with personal knowledge of patients to co-ordinate the contact tracing was a key factor in the high success rate for screening. The principle of a single individual with general responsibility for managing a contact group is a cost effective approach.

\section{Recommendations}

One case of tuberculosis was discovered equating to a cost of $£ 44$ per contact screened or $£ 25000$ per case detected. Two cases of lung cancer were diagnosed earlier than if screening had not occurred and staff members who had not received BCG were identified. The occupational health department has changed its policy to conform with the latest BTS guidelines and closer liaison and regular policy review are planned. The Trust has reinforced the importance of attendance for screening in any outbreak situation.

However, guidelines are promulgated in part on the basis that most cases of clinical tuberculosis are due to reactivation of earlier infection. Two recent reports have suggested that $30-40 \%$ of infections recorded in New York City and San Francisco during a two year period were due to recent infection. ${ }^{910}$ Small et $a l^{9}$ described one non-compliant individual who, on the basis of restriction fragment length polymorphism (RFLP) typing, infected $6 \%$ of all identified cases in San Francisco despite the efforts of an excellent tuberculosis control programme which ensured that $95 \%$ of patients completed treatment during the study period. Nosocomial spread of $M$ tuberculosis infection in a renal transplant unit in the USA in which five patients died was recently proved using RFLP analysis, ${ }^{11}$ and the same technique was used to disprove a nosocomial outbreak in a London renal unit. ${ }^{12}$ Molecular techniques are likely to be of increasing value in analysing nosocomial as well as community outbreaks. ${ }^{14}$ If these findings are generally applicable then contact tracing with molecular analysis of positive cultures may become more common.

In a similar incident the 1994 BTS guidelines, ${ }^{7}$ which have superseded the 1990 guidelines, ${ }^{6}$ should form the basis for its investigation, supplemented by the following recommendations:

(1) clear executive responsibility for the in- cident must be established from the outset;

(2) adequate financial resources from central Trust/hospital funds must be identified from the outset;

(3) a small task force must be established with staff assigned full time as required. The group should have sufficient delegated authority from the EICC to conduct all actions needed to deal with the incident;

(4) it must be seen as a management priority that accurate lists of contacts should be provided;

(5) all possible efforts must be made to identify deceased patients to ascertain whether death was related to tuberculosis and to prevent distress to families inadvertently receiving notifications for screening;

(6) patients and GPs must be fully informed of the incident, the risk of infection and likely treatment needed, and must have ready access to further information;

(7) patients and GPs must be informed of the conclusions of individual investigations and when the follow up period is terminated;

(8) staff confidentiality must be preserved on ethical grounds and to encourage staff to come forward;

(9) monitoring, data collection, and analysis must be standardised;

(10) local guidelines should be formulated so that an action plan is available that can be instituted quickly;

(11) staff contacts must be aware that they have a responsibility to attend for screening and senior line managers must make time available regardless of other commitments for staff to attend appointments with occupational health or designated medical or nursing staff.

The authors thank the staff of the renal unit, particularly Ms Monica McSharry and Professor B Hendry, Professor J Moxham and Dr B Gray, and Ms Kathy Watkins, Ms Ann Doherty, Mr Jamil Choglay and Ms Linda Creegan for their help and support in the conduct of this investigation.

1 Rodrigues LC, Smith PG. Tuberculosis in developing countries and methods for its control. Trans $R$ Soc Trop Med Hyg 1990;84:739-44.

2 Bloom BR, Murray CJL. Tuberculosis: commentary on a re-emergent killer. Science 1992;257:1055-64.

3 Mitwali A. Tuberculosis in patients on maintenance dialysis. Am $\mathcal{F}$ Kidney Dialysis 1991;18:579-82.

4 Higgins RM, Cahn AP, Porter D, Richardson AJ, Mitchell RG, Hopkin JM, et al. Mycobacterial infections after renal transplantation. $Q \mathcal{F}$ Med 1991;78:145-53.

5 Qunibi WY, al-Sibai MB, Taher S, Harder EJ, de Vol E, al-Furayh $\mathrm{O}$, et al. Mycobacterial infections after renal transplantation - report of 14 cases and review of the literature. $Q \mathcal{F}$ Med 1990;77:1039-60.

6 Joint Tuberculosis Committee of the British Thoracic Society. Control and prevention of tuberculosis in the United Kingdom: Code of Practice 1994. Thorax 1994;49:1193200.

8 NHS Management Executive. Public health: responsibilities of the NHS and the roles of others. HSG (93) 56, 1993. 9 Small PM, Hopewell PC, Singh SP, Paz A, Parsonnet J, Ruston DC, et al. The epidemiology of tuberculosis in San Francisco - a population-based study using conventional and molecular methods. $N$ Engl $₹$ Med 1994; 330: 1703-9.

10 Alland D, Kalkut GE, Moss AR, McAdam RA, Hahn JA, Bosworth W, et al. Transmission of tuberculosis in New Bosworth W, et al. Transmission of tuberculosis in New
York City - an analysis by DNA fingerprinting and conYork City - an analysis by DNA fingerprinting and con-
ventional epidemiological methods. $N$ Engl $\mathcal{F}$ Med 1994;
$330: 1710-6$.

11 Jereb JA, Burwen DR, Dooley SW, Haas WH, Crawford 
JT, Geiter LJ, et al. Nosocomial outbreak of tuberculosis in a renal transplant unit: application of a new technique for restriction fragment length polymorphism analysis of Mycobacterium tuberculosis isolates. $\mathcal{F}$ Infect Dis 1993;168: Mycobacterin
$1219-24$.

12 Bendall RP, Drobniewski FA, Jayasena D, Nye PM, Uttley AHC, Scott GM. Restriction fragment length polymorphism analysis rules out cross-infection among rena patients with tuberculosis. $\mathcal{F}$ Hosp Infect 1995 (in press).
13 Drobniewski FA, Bendall R, Nye P, Jayasena S, Scott G, Uttley A. Use of restriction fragment length polymorphism (RFLP) analysis of Mycobacterium tuberculosis to rule out cross-infection on a renal unit. Proceedings of the $3 r d$ International Conference of the Hospital Infection Society, London 1994, abstract F8/2.

14 Drobniewski FA, Kent RJ, Stoker NG, Uttley AHC. Molecular biology in the diagnosis and epidemiology of tuberculosis. $\mathcal{F}$ Hosp Infect 1994;28:249-63. 